\title{
El ahorro de energía por adiciones al cemento
}

P. DUTRON, Director del Centre National de Recherches Scientifiques et Techniques pour I'Industrie Cimentière (C.R.I.C.)

\section{INTRODUCCION}

Antes de tratar un tema, cualquiera que sea, conviene ponerse de acuerdo previamente sobre el sentido que se da a las palabras empleadas. A lo largo de esta exposición, consideraremos que hay o que puede haber en un cemento:

- Un componente principal, el clínker portland, que se encuentra en mayor o menor proporción en todos los cementos clásicos dignos de este nombre.

- Uno o varios componentes secundarios, que pueden repartirse en dos categorías según que su actividad preponderante sea:

- de naturaleza química, a saber:

- la escoria de horno alto,

- la puzolana natural o artificial,

- las cenizas volantes, a menudo incluidas en las puzolanas;

- de naturaleza física, como:

- los polvos minerales.

- Uno o varios aditivos, a saber:

- el sulfato de calcio, que actúa, en una u otra de sus formas, como regulador de fraguado ;

- los agentes de molienda, que facilitan esta operación especialmente costosa;

- los aditivos, que proporcionan al cemento, y por lo tanto al hormigón, una propiedad especial tales como la oclusión de aire, la aceleración de endurecimiento, la hi.drofugación, ...

A continuación se tratarán los componentes secundarios:

De forma esquemática y global el problema que puede plantear el empleo de estos componentes queda resuelto:

- reglamentariamente, si el cemento considerado está autorizado por las normas y demás reglamentos nacionales y/o internacionales; 
- técnicamente, si se tiene toda seguridad de que el cemento con componentes secundarios permitirá la fabricación de hormigón de calidad a corto y a largo plazo;

- económicamente, si este cemento tiene un precio de costo técnico de un nivel reducido y si al mismo tiempo el precio de venta ha podido mantenerse a un nivel razonable, teniendo en cuenta el valor de satisfacción que ofrece el producto al cliente.

No desarrollaré, desde luego, este punto de vista económico que no me concierne y voy a tratar sucesivamente los dos primeros aspectos.

\section{DESCRIPCION DE LA SITUACION ACTUAL}

En el plano de los hechos, veamos, en primer lugar, cuáles son los tipos de cemento que están actualmente normalizados y cuál es su valor representativo en la producción.

La tabla I expone la situación en los 9 países de la C.E.E. y en España. Para aclarar la situación, los cementos se distribuyen en cuatro categorías:

- los cementos portland puros;

- los cementos portland compuestos con uno o varios componentes secundarios, cuyo contenido máximo se encuentra aproximadamente en un $35 \%$ (en la práctica de 30 a $40 \%$ según los países);

- los cementos clínker-escoria, cuyo contenido de escoria es superior al $35 \%$;

- los cementos clínker-puzolana, que deben responder al ensayo de puzolanicidad.

Para las tres últimas categorías, la tabla I da los límites de contenidos de componentes secundarios permitidos por las normas. Siempre que es posible, se indica el valor que representa en la producción nacional el tipo de cemento considerado.

Sin entrar en detalles, se observa cuánto varía la situación de un país a otro; en parte, por circunstancias y disponibilidades locales, en parte, por tradición:

- ciertos países (Dinamarca, Irlanda y Gran Bretaña) sólo producen, prácticamente, cemento portand;

- otros (Bélgica y Alemania) producen $3 / 4$ de portland y $1 / 4$ de cemento clínker-escoria ;

- en Italia y en Holanda, el cemento portland sólo representa alrededor de la mitad de la producción (el 55 y el $40 \%$ ), siendo esencialmente el resto de cemento puzolánico en Italia y de cemento clínker-escoria en Holanda;

- en Francia, la parte que ocupa el cemento portland puro apenas sobrepasa 1/4 de la producción, siendo la casi totalidad del resto los cementos portland compuestos, ocupando un lugar relativamente pequeño los cementos con gran contenido de escorias;

- finalmente, en el extremo del abanico se halla Luxemburgo que sólo produce cementos clínker-escoria, siendo elevado la mayoría de las veces el contenido de escoria; por supuesto se debe tener en cuenta la importancia de la industria siderúrgica en este país. 


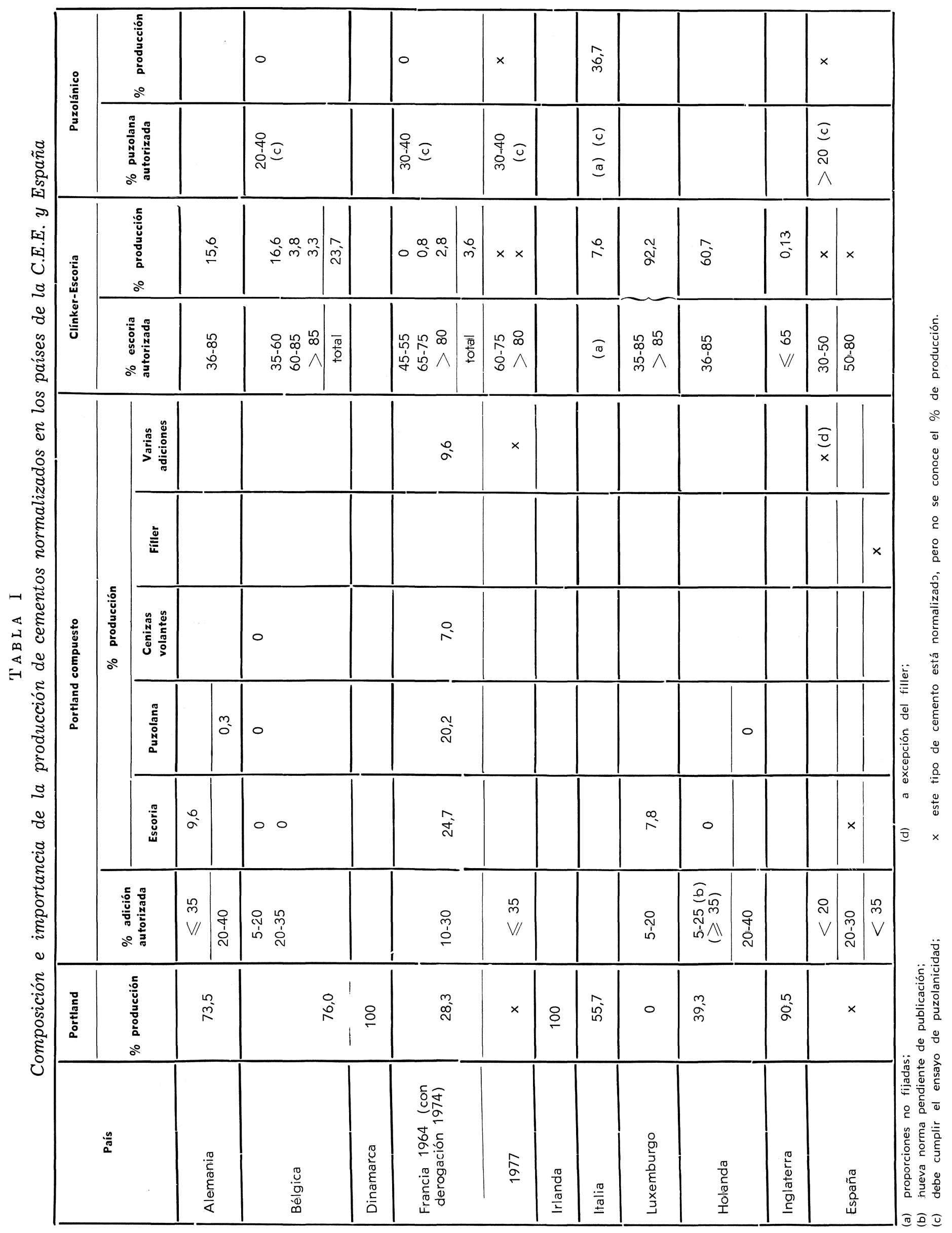




\title{
3. PERSPECTIVAS DE EVOLUCION
}

Desde luego sería presuntuoso pretender dar una respuesta clara a semejante cuestión.

Digamos que es lógico pensar que, teniendo en cuenta el coste de la caloría y la preocupación de cada país por reducir al mínimo su dependencia frente a fuentes extranjeras de suministro de energía, se asistirá a una presión tanto económica como política para que una parte tan elevada como sea posible de los cementos portland puros sea sustituida por cementos portland compuestos en la medida en que disponga cada país o región de componentes secundarios adecuados.

\section{ELIMINACION DE LOS OBSTACULOS REGLAMENTARIOS}

Para prepararse a esta probable evolución conviene eliminar los obstáculos reglamentarios, tales como la ausencia de ciertos tipos de cementos en las normas nacionales. Digamos, a este respecto, que la nueva norma española es irreprochable, ya que prevé todas las composiciones de cemento razonablemente imaginables; podrá servir de guía para el Grupo de Trabajo 51 "Cementos" del Comité Europeo de Normalización (CEN) que debería conseguir una norma europea que defina los diversos tipos de cemento.

\section{ELECCION Y CRITERIOS DE CALIDAD DE LOS COMPONENTES SECUNDARIOS. CONTENIDOS MAXIMOS ADMISIBLES EN ESTOS}

\begin{abstract}
Abierta así la vía reglamentaria, conviene, en el plano técnico, definir, para cada posible componente secundario, los criterios a los que debe responder y la proporción que se puede introducir en mezcla con el clínker sin perjudicar las calidades de los hormigones que se fabriquen con tales cementos.
\end{abstract}

\subsection{Escoria}

Este componente, como se sabe, ya está ampliamente normalizado y abundantemente utilizado, especialmente en la parte occidental del continente europeo.

La larga experiencia que se tiene de los cementos a base de escoria explica y justifica que las normas autoricen su empleo hasta porcentajes sumamente elevados $(>85 \%)$, reservados sin embargo, en este caso, a los cementos destinados a usos relativamente especiales.

Lo que ha limitado probablemente hasta hoy el empleo de la escoria se debe: bien al hecho de que ciertos países no la producen, bien al hecho de que en los países que la producen estaba reservada hasta ahora a otros usos, o bien a que a veces no reúne las características exigidas por las condiciones de obtener la fundición.

No es por eso menos cierto que la escoria ha adquirido desde hace mucho tiempo un derecho de ciudadanía indiscutible. 


\subsection{Puzolanas naturales $y$ artificiales}

Se trata en primerísimo lugar de las puzolanas naturales que la mayoría de los países mediterráneos utilizan desde hace tiempo en una proporción que suele oscilar entre el 20 y el $40 \%$. Como en el caso de la escoria, la experiencia es amplia y no merece la pena extenderse a este respecto.

Señalemos, sin embargo, como se verá más adelante, que la calidad de puzolana se comprueba, o químicamente apreciando la cantidad de cal fijada por la puzolana como en el ensayo de puzolanicidad que nos viene de Italia, objeto de una recomendación ISO y que constituirá pronto una norma europea; o bien mecánicamente comparando la evolución de las resistencias de un mortero de cemento clínker-puzolana con las de un mortero de cemento portland.

Sin dejar el campo de los productos naturales, debe prestarse una especial atención al interés que podría ofrecer la reactivación por tratamiento térmico de ciertas arcillas que adquieren de este modo un excelente carácter puzolánico; el desarrollo del empleo de tales componentes depende esencialmente de la ventaja económica que se pueda lograr.

El tercer tipo de material puzolánico que hay que tomar en consideración es la ceniza volante. Se comprueba que este material se cita muy pocas veces en las normas como componente del cemento y que aún se utiliza muy poco en la producción industrial del cəmento.

Los porcentajes máximos autorizados en las normas son del mismo orden de magnitud que en caso de la puzolana natural.

Sin embargo, se trata de un material que se dispone en cantidad en los países que utilizan el carbón como combustible en las centrales térmicas.

Un obstáculo en su desarrollo radica, sin ninguna duda, en el hecho de que las calidades de las cenizas volantes pueden con frecuencia ser bastante variables; esto no impide que la reticencia actual por emplear la ceniza volante en la fabricación del cemento, que proviene de una laguna de normas o de una posición deliberada de la industria de cemento, trae como consecuencia que en muchos países se introduzcan cenizas volantes en los hormigones en detrimento del contenido de cemento de los mismos.

Esto se ha manifestado claramente en la reunión del Grupo de Estudio de Ensayos del Cemento y del Hormigón del Cembureau, en la que se ha señalado que, cuando las normas relativas al hormigón fijan en un nivel bastante bajo el contenido mínimo de cemento, por ejemplo $250 \mathrm{~kg} / \mathrm{m}^{3}$, se brinda así la ocasión a los usuarios de añadir, a estos 250 $\mathrm{kg}$ de cemento $50 \mathrm{~kg}$ de cenizas volantes para conseguir, en suma, en condiciones menos buenas, un cemento portland que contiene un $16 \%$ de cenizas volantes.

Todo esto justifica que se estudie con mayor detalle este problema. Con un breve estudio bibliográfico se han podido preparar las tablas II y III que resumen las especificaciones de cierto número de normas que dan las características físicas y químicas de las puzolanas y de las cenizas, por una parte (tabla II), y los criterios con los que se puede determinar el grado de su actividad puzolánica, por la otra (tabla III). Recordaremos, muy especialmente, las exigencias en materia de:

- contenido de agua: una ceniza volante que se tenga que introducir en el molino con el clínker debe estar por supuesto prácticamente seca;

- pérdida al fuego: la presencia de inquemados en cantidad excesiva puede dar origen a diversas anomalías $y$, sobre todo, a aparición en la superficie de partículas negruzcas que dañan el aspecto del hormigón; parece ser que, a este respecto, ciertos porcentajes máximos autorizados son demasiado elevados. 
T A B L A II

Criterios de calidad físicos y químicos que se requieren para los componentes secundarios de los cementos

\begin{tabular}{|c|c|c|c|c|c|}
\hline País & Alemania & Occidental & Aus & & Inglaterra \\
\hline Norma & (a) & DIN 51043 & önorm B 3323 & önorm B 3319 & $\begin{array}{l}\text { BS } 3892: 1965 \\
\text { (para hormigón) }\end{array}$ \\
\hline $\begin{array}{l}\text { Naturaleza del } \\
\text { componente }\end{array}$ & Cenizas volantes & Trass & Trass & Cenizas volantes & Cenizas volantes \\
\hline Finura & $\geqslant 3.000 \mathrm{~cm}^{2} / \mathrm{g}$ & $\begin{array}{l}\text { máx. } 20 \%> \\
>200 \mu m\end{array}$ & $\begin{array}{c}\text { máx. } 10 \%> \\
>90 \mu \mathrm{m}\end{array}$ & - & $\begin{array}{l}\text { A: } 1.250 \text { a } 2.750 \mathrm{~cm}^{2} / \mathrm{g} \\
\text { B: } 2.750 \text { a } 4.250 \mathrm{~cm}^{2} / \mathrm{g} \\
\text { C: }>4.250 \mathrm{~cm}^{2} / \mathrm{g}\end{array}$ \\
\hline $\begin{array}{c}\text { Conitenido de } \\
\text { agua }(\%)\end{array}$ & & $\begin{array}{c}>6 \text { (agua } \\
\text { unida } \\
\text { químicamente) }\end{array}$ & - & - & $\leqslant 1,5$ \\
\hline $\begin{array}{l}\text { Pérdida al fue- } \\
\text { go }(\%)\end{array}$ & $(\leqslant 5)$ & - & - & $\leqslant 7$ & $\leqslant 7$ \\
\hline $\mathrm{SO}_{3} \quad(\%)$ & $(\leqslant 3)$ & - & $\leqslant 1$ & $\leqslant 3,5$ & $\begin{array}{l}\leqslant 1,5 \text { ó } 2,5 \\
\text { (según finura) }\end{array}$ \\
\hline $\begin{array}{l}\mathrm{SiO}_{2}+\mathrm{Al}_{2} \mathrm{O}_{3}+ \\
+\mathrm{Fe}_{2} \mathrm{O}_{3}(\%)\end{array}$ & & - & - & - & - \\
\hline $\mathrm{CaO}(\%)$ & 2 a. 6,5 & - & $\leqslant 3$ & - & - \\
\hline $\mathrm{MgO}(\%)$ & 2 a 3 & - & $\leqslant 1$ & - & $\leqslant 4$ \\
\hline $\mathrm{SiO}_{2} / \mathrm{R}_{2} \mathrm{O}_{3}$ & . & 一 & $\geqslant 2$ & - & - \\
\hline $\begin{array}{l}\text { Alcalis } \\
\left(\% \quad \mathrm{Na}_{2} \mathrm{O}\right)\end{array}$ & $\vdots$ & & & & \\
\hline
\end{tabular}

(a) Se trata de exigencias no normalizadas que hay que respetar para conseguir una marca de calidad que autorice el empleo de cenizas como adición al cemento (máx. $20 \%$ del peso del cemento) en el hormigón; los 2 valores extremos ( ) están garantizados por el producto comercial «Efa-füller».

T A в L A II (bis)

Criterios de calidad físicos y químicos que se requieren para los componentes secundarios de los cementos

\begin{tabular}{|c|c|c|c|c|c|}
\hline País & Turquía & \multicolumn{3}{|c|}{ Estados Unidọs } & Alemania Oriental \\
\hline Norma & TS 639 (1968) & \multicolumn{3}{|c|}{$\begin{array}{c}\text { ASTM C } 618-73 \\
\text { (para hormigón) }\end{array}$} & TGL $28101 / 03$ \\
\hline \multirow{2}{*}{$\begin{array}{l}\text { Naturaleza del } \\
\text { componente }\end{array}$} & \multirow{2}{*}{ Cenizas volantes } & Cenizas volantes & \multicolumn{2}{|c|}{ Puzolanas } & \multirow{2}{*}{ Cenizas volantes } \\
\hline & & 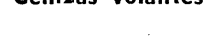 & Naturales & Artificiales & \\
\hline Finura & 一 & $\begin{array}{l}>6.500 \\
\mathrm{~cm}^{2} / \mathrm{cm}^{3}\end{array}$ & $\begin{array}{l}>12.000 \\
\mathrm{am}^{2} / \mathrm{cm}^{3}\end{array}$ & $\begin{array}{l}>6.500 \\
\mathrm{~cm}^{2} / \mathrm{cm}^{3}\end{array}$ & - \\
\hline $\begin{array}{l}\text { Contenido de } \\
\text { agua }(\%)\end{array}$ & $\leqslant 3$ & $\leqslant 3$ & $\leqslant 3$ & $\leqslant 3$ & \\
\hline $\begin{array}{l}\text { Pérdida al fue- } \\
\text { go }(\%)\end{array}$ & $\leqslant 10$ & $\leqslant 12$ & $\leqslant 10$ & $\leqslant 10$ & $\leqslant 15$ \\
\hline $\mathrm{SO}_{3}(\%)$ & $\leqslant 5$ & $\leqslant 5$ & $\leqslant 4$ & $\leqslant 4$ & $\leqslant$ \\
\hline $\begin{array}{l}\mathrm{SiO}_{2}+\mathrm{Al}_{2} \mathrm{O}_{3}+ \\
+\mathrm{Fe}_{2} \mathrm{O}_{3}(\%)\end{array}$ & $\geqslant 70$ & $\geqslant 85$ & $\geqslant 75$ & $\geqslant 75$ & \\
\hline $\mathrm{CaO}(\%)$ & $\leqslant 6$ & - & - & - & \\
\hline $\mathrm{MgO}(\%)$ & $\leqslant 5$ & - & $\leqslant 5$ & $\leqslant 5$ & \\
\hline $\mathrm{SiO}_{2} / \mathrm{R}_{2} \mathrm{O}_{3}$ & - & - & - & - & \\
\hline $\begin{array}{l}\text { Alcalis } \\
(\% \quad \mathrm{Na} 2 \mathrm{O})\end{array}$ & & $\leqslant 1,5$ & (a petic & $\underset{\text { especial) }}{\leqslant} 1,5$ & \\
\hline
\end{tabular}


T A B L A III

Criterios para apreciar la actividad puzolánica de los componentes secundarios de los cementos

\begin{tabular}{|c|c|c|c|c|}
\hline País & \multicolumn{2}{|c|}{ Alemania Occidental } & \multicolumn{2}{|c|}{ Austria } \\
\hline Norma & (y) & DIN 51043 & Önorm B 3323 & önorm B 3319 \\
\hline $\begin{array}{l}\text { Naturaleza del } \\
\text { componente }\end{array}$ & Cenizas volantes & Trass & Trass & Cenizas volantes \\
\hline $\begin{array}{l}\text { Composición del o } \\
\text { de los morteros }\end{array}$ & $\begin{array}{l}2 \text { morteros nor- } \\
\text { males con: } \\
\text { a: cemento: } 1 \\
\text { b: cemento: } 0,8 \\
\text { cenizas: } 0,2\end{array}$ & $\begin{array}{l}\text { Trass }: 1 \\
\text { Cal hidratada: } 0,8 \\
\text { Arena normal: } 1,5\end{array}$ & $\begin{array}{ll}\text { Trass } & : 0,7 \\
\text { Cal hidratada: } & 0,3 \\
\text { Arena normal: } & 3,0\end{array}$ & $\begin{array}{l}3 \text { morteros nor- } \\
\text { males con: } \\
\text { a: cemento puro } \\
\text { b: } 0,85 \text { cemento } \\
0,15 \text { cenizas } \\
\text { c: } 0,85 \text { cemento } \\
0,15 \text { arena fina }\end{array}$ \\
\hline $\begin{array}{l}\text { Resistencia a la } \\
\text { compresión } \\
\left(\mathrm{kp} / \mathrm{cm}^{2}\right)\end{array}$ & & $\begin{aligned} 7 \text { días: } & >45 \\
28 \text { días: } & >140\end{aligned}$ & $\begin{array}{c}- \\
28 \text { días: }>50\end{array}$ & \\
\hline $\begin{array}{l}\text { Relación de la re- } \\
\text { sistencia e n tre } \\
\text { morte ros con } \\
\text { adición y morte- } \\
\text { ro-testigo }(\%)\end{array}$ & $\begin{array}{l}\frac{b}{a} \geqslant 90 \\
a 2,7 \text { y } 28 \text { días }\end{array}$ & & & $\begin{array}{l}\frac{b-c}{a-c} \geqslant 30 \\
\text { a } 28 \text { días }\end{array}$ \\
\hline
\end{tabular}

(y) Se trata de exigencias no normalizadas que hay que respetar para conseguir una marca de calidad que autorice el empleo de cenizas como adición al cemento (máx. $20 \%$ del peso de cemento) en el hormigón; los 2 valiores extremos ( ) están garantizados por el producto comercial «Efa-füller».

\section{T А в L A III (bis)}

Criterios de apreciación de la actividad puzolánica de los componentes secundarios de los cementos

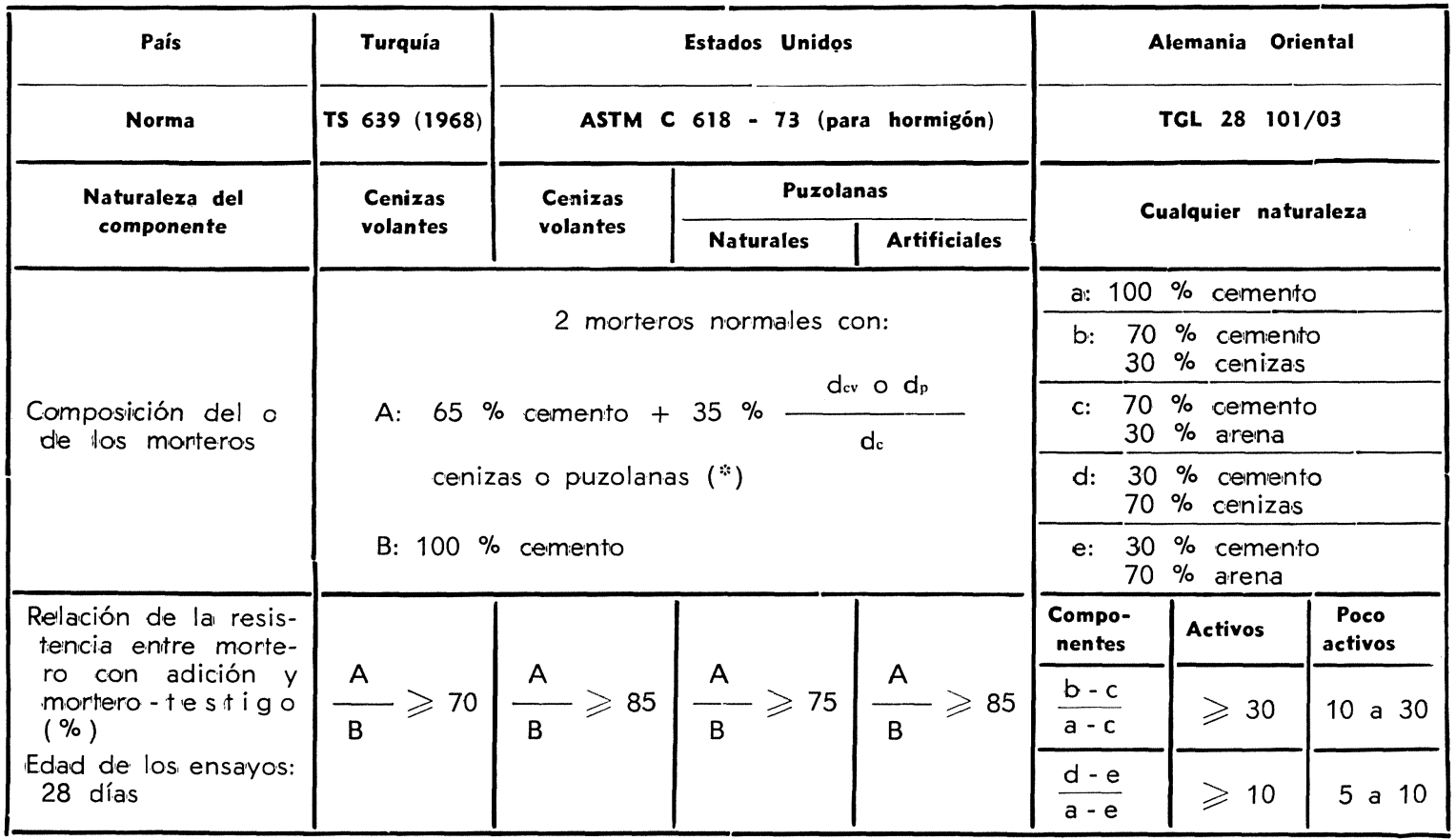

(*) $\mathrm{d}_{c v}, \mathrm{~d}_{p} \circ \mathrm{d}_{c}=$ densidad de las cenizas volantes, puzolanas o cemento. 


\subsection{Polvos minerales}

Por este nombre se entienden unos fillers que proceden de rocas molidas, la mayoría de las veces de tipo calizo y en ocasiones silíceas. Apenas se encuentra en la literatura, ni en las normas, indicaciones sobre los criterios a los cuales deberían responder tales materiales.

La contribución que se les exige no es la de poseer propiedades puzolánicas ni hidráulicas como la escoria; su papel es más bien físico, pues pu€den mejorar las características reológicas de la pasta de cemento.

Por otra parte se comprueba que han aparecido, en las normas de varios países, cementos en los que existe una pequeña proporción de adición a los cementos portland conservando su calificación de "puro", estando limitada esta adición al $3 \%$ en Francia y en Suecia y al $5 \%$ en Bélgica con el vocablo de "materias tintóreas" (*).

Otros países han llegado más lejos, como Finlandia donde el cemento portland puede contener hasta el $15 \%$ de fíller o de otros componentes secundarios sin cambiar de denominación, y sobre todo Alemania del Este y Francia, cuyo cemento portland compuesto CPJ puede contener como máximo, respectivamente, hasta 20 y $35 \%$ de componentes secundarios, entre los cuales se encuentra el fíller.

La nueva norma española ha entrado también en esta vía, creando esta categoría de cemento, aunque prohibiendo en cambio tales adiciones en el cemento portland puro.

\section{CALIDAD DE LOS HORMIGONES FABRICADOS CON UN CEMENTO PORTLAND COMPUESTO}

Para estar tranquilos respecto al empleo de un cemento portland compuesto en los hormigones, no basta por supuesto que el cemento responda a todas las prescripciones que figuran en la norma "Cemento". Es preciso además que el hormigón que se fabrique con este cemento presente, desde todos los puntos de vista, las mismas garantías que si se hubiera fabricado por ejemplo con un cemento portland tradicional.

La norma ASTM C 465-74 da una respuesta parcial a este problema; prevé, efectivamente, seguir la evolución de la retracción y de las resistencias en el mortero o en el hormigón.

Sin embargo, resulta que las comprobaciones del buen comportamiento del hormigón deberían ampliarse a todo lo que afecta a su durabilidad, especialmente en presencia de los agentes atmosféricos; se piensa aquí en la resistencia a los ciclos humedad-sequedad y hielo-deshielo que por supuesto está relacionada con la capacidad de absorción de agua por capilaridad y por inmersión del hormigón y de la pasta de cemento.

Tales estudios profundos resultarían indispensables si se quisiese o se debiese un día determinar el límite extremo de adición de componentes secundarios en el cemento.

(*) Traducción de la versión original «matières tinctzriales*. 


\section{\%. CONCLUSIONES}

La maquinaria moderna de las fábricas de cemento permite hoy día, casi inevitablemente, fabricar clínker que dé cementos portland con muy buenas cualidades mecánicas; la finura de un cemento no puede ser demasiado grosera, so pena que dañe las propiedades de la pasta de cemento en materia de plasticidad y de untuosidad.

Las cualidades de tales cementos son frecuentemente más elevadas de lo que requiere el uso que se va a hacer de ellos.

Aparte de las consideraciones energéticas e incluso simplemente económicas, la técnica muestra que existe un gran campo para que los cementos compuestos de un clínker de alta calidad y de componentes secundarios más o menos activos o inertes químicamente, molidos a una finura suficientemente grande, den una pasta reológicamente satisfactoria.

Una vez que la economía y la técnica se encuentran. valía la pena tenerlo en cuenta. 
1. Lo presidió el Sr. NADAL y lo abrió el Sr. AGUANEL, quien quiso saber, en primer lugar, si la caliza como adición, de la que el conferenciante no había hablado, era activa o no; en segundo lugar, en qué consistía y qué efectos tenía la formación de carboaluminatos; y en tercer lugar, qué influencia tenían los fillers calizos en la reología del hormigón fresco y en la impermeabilidad del hormigón endurecido.

Respondió el Sr. DUTRON que en su exposición había situado la caliza entre los constituyentes secundarios cuya acción preponderante es física, a los cuales se les llama polvos minerales en Bélgica - y fillers en otras partes-, y que, en general, son calizos o silícicos. Y que suponía que el Sr. AGUANEL se refería a la caliza finamente molida.

Este preguntó si esta caliza daba lugar a reacciones químicas en la pasta de cemento.

El Sr. DUTRON dijo que cuando se piensa en añadir caliza molida al cemento él cree que se hace por aportar mejoras físicas, y no por mejorar a base de las combinaciones tales como los carboaluminatos que se pudieran formar. Lo que no quiere decir -añadió-, que no tengan lugar acciones químicas; simplemente que no se piensa en ellas cuando se eligen tales adiciones.

El Sr. AGUANEL insistió preguntando si el conferenciante había comprobado que un cemento con fíller calizo daba hormigones de mayor impermeabilidad por formación de carboaluminatos, a lo que el Sr. DUTRON contestó que no lo había estudiado, porque en su país no empleaban hasta el momento adiciones calizas. Que al respecto piensan sólo en economías, que es lo que se persigue con tales adiciones y que están comenzando a estudiar ahora la porosidad de los hormigones en general, en función de cementos conteniendo fillers o no, pero que no podía decir más.

Sin abandonar el tema el Sr. AGUANEL preguntó, por último, si el Sr. DUTRON creía que la adición de un poco de caliza mejoraba al cemento. Respondió éste que desde un punto de vista físico indiscutiblemente sí, y que hasta diría que en una proporción aproximada del $10 \%$. El Sr. AGUANEL agradeció esta respuesta.

2. A continuación el Sr. FRANCO, de Colombia, preguntó si no había incompatibilidad entre dos adiciones simultáneas, por ejemplo, ceniza volante y puzolana natural.

El Sr. DUTRON dijo que no lo sabía, porque en Bélgica se carece de experiencia sobre ello. Pero que creía no equivocarse al decir que incluso en Francia, país más avanzado al respecto, no se han hecho cementos ternarios con cenizas y puzolanas. Lo que sí sabía. por la bibliografía y por conversaciones con los especialistas del CERILH francés, es que los cementos ternarios a base de escorias y cenizas, o de escorias y puzolanas, constituyen "un buen cocktail", porque ambas adiciones tienen en cada caso acciones que se compensan y complementan, con el resultado de un efecto beneficioso.

3. El Sr. CALLEJA preguntó después la opinión del Sr. DUTRON acerca de las adiciones de tipo silícico, de las que éste no había hablado. ¿Qué efecto creía que ejercían sobre el cemento portland, tanto desde el punto de vista físico como químico? 
Respondió el Sr. DUTRON que no lo sabía; que hablar de sílice es muy general, ya que la hay activa e inactiva. Pero si se toma en el sentido de áridos silícicos para hormigón, los cuales se muelen para obtener un polvo fino, diría que el polvo de áridos calcáreos es mejor que el de los silícicos, ya que la caliza y el cemento "se cocinan" bien juntos, por jugar un papel en ello la epitaxia que da cohesión a la pasta y al mortero, y por lo tanto contribuye a la resistencia a la tracción. Esto no sucede con la sílice corriente, si bien los materiales silícicos activos muy finos - ultrapuros- dan resultados extraordinarios, aunque no son explotables industrialmente, por ser muy caros según él creía.

El Sr. CALLEJA dijo que el Sr. DUTRON había pronunciado precisamente la palabra que él había querido escuchar: epitaxia.

4. A continuación el Sr. ULLOA preguntó por los efectos benéficos en los aspectos físicos y químicos de las cenizas volantes.

Le respondió el Sr. DUTRON diciendo que las cenizas se emplean, ante todo, por razones económicas. Añadió que de lo dicho por él en su exposición convenía destacar que el cemento portland puro es un mal cemento. Muy bueno en muchos aspectos, porque así lo ha probado, pero es un cemento de ricos, y que en Europa ya no lo éramos tanto. Por ello - dijo-, se lucha económicamente por sustituirlo más o menos bien para obtener, no obstante, un producto de calidad. Ese es el primer objetivo de las cenizas. Por otra parte, químicamente y en la medida en que tienen propiedades y actividad puzolánica fijando cal, las hay dignas de figurar en plano de igualdad con las puzolanas naturales, confiriendo características químicas de resistencia a las aguas agresivas, nada despreciables. Añadió el Sr. DUTRON que las cenizas se utilizaban en Bélgica en gran escala como materia prima en el horno de cemento, y que en este aspecto no molestaba su pérdida al fuego - los inquemados-; por el contrario — dijo-, si se utilizan en hornos, cuanto más inquemados contengan más se ahorra por el aprovechamiento de la fracción última de carbón que contienen. Además —añadió-, trabajando en vía húmeda (que todavía abunda) se pueden utilizar sin secar, resultando entonces gratuitas. Por el contrario -concluyó-, las que se añaden en el molino deberían tener pocos inquemados -menos de $2 \%$, lo cual es utópico-, y en todo caso menos de $5 \%$.

5. El Sr. SOLER preguntó después acerca de la molienda conjunta o por separado de las adiciones activas.

El Sr. DUTRON dijo que no creía equivocarse al decir que en la mayoría de los casos se efectuaba la molienda conjunta y que, en general, así ocurría con los cementos binarios clásicos.

6. El Sr. FERNANDEZ MOLINA solicitó el punto de vista del conferenciante acerca de la trabajabilidad del hormigón fresco con cenizas contenidas en el cemento, o añadidas a la hormigonera en obra, si bien ya sabía que desde el punto de vista de la resistencia del hormigón endurecido era mejor que las cenizas estuviesen contenidas ya en el cemento.

El Sr. DUTRON respondió que, a igualdad de todo lo demás, la trabajabilidad debía de ser la misma, tanto si la ceniza se añadía en fábrica o en obra. Pero que en obra la homogeneidad de mezcla podía ser menor, por lo difícil que resulta en ella mezclar íntimamente polvos finos; y que, en todo caso, en obra se dependía de la disponibilidad y suministros. A escala industrial - dijo-, conviene tener un gran almacén que garantice más la homogeneidad, con lo cual se gana en regularidad de la trabajabilidad, lo cual es importante y menos peligroso que tener que actuar quitando o poniendo agua para mantener una trabajabilidad determinada. Añadió que en el CEB (Comité Europeo del Hormigón), donde se habla de resistencia característica en la que juega la dispersión -o la desviación estándar-, tal argumento es el máximo para utilizar un producto industrial y no foráneo, por llamarlo así. 
\%. A continuación el Sr. PEREZ ZUBIZARRETA planteó la cuestión del empleo de las adiciones aún sin medir el aspecto del ahorro de combustible. Dijo que el Sr. DUTRON había hablado de las ventajas de los cementos con constituyentes secundarios, y que él querría saber si sus opiniones al respecto serían las mismas de no estar de por medio la crisis del petróleo de 1973.

El Sr. DUTRON dijo que creía que lo que se planteaba era una cuestión económica en estos términos: si fuese cierto que, incluso a igualdad de calidad, un cemento portland compuesto con $25 \%$ de escoria - por ejemplo, y por ser un caso conocido-, cuesta más que un cemento portland puro porque la energía para la clínkerización es barata, o porque la escoria escasea o se emplea para otros fines, se fabricaría y utilizaría cemento portland puro y no se pensaría en el de escorias. La fabricación y el uso de uno u otro no depende de modas o caprichos, sino de razones y necesidades económicas, dentro de hacer en todo caso productos técnicamente buenos, lo cual es tarea de los Institutos de Investigación.

8. El Sr. AGUANEL dijo no estar del todo de acuerdo, porque muchas veces los cementos con escorias son precisos para una obra hidráulica o resistente a una determinada agresividad, y entonces se fabrican no sólo por razones económicas, sino también por necesidades técnicas.

El Sr. DUTRON se manifestó conforme con ello, pero en tales casos - dijo-, la proporción de escoria en los cementos se sale bastante de la que corresponde a los que se llaman cementos portland compuestos, ya que, por ejemplo, los cementos de horno alto resistentes a las acciones agresivas deben contener, por lo menos, del orden del $70 \%$, según es corriente en muchas normas. Entonces, y no ignorando la crisis de la energía, es necesario hacer tales cementos, aunque también pueda haber cementos portland especiales capaces de resistir a los sulfatos, como ha puesto de relieve el Sr. CALLEJA en un estudio comparativo de normas.

9. Finalmente el Sr. CALLEJA hizo unos comentarios a dos cuestiones previamente planteadas. Una - dijo-, es la relativa a la molienda conjunta o por separado del clínker y de las adiciones. En efecto, la tendencia hasta el momento, que parece seguir manteniéndose, es hacia la molienda conjunta. Razones: inercia, costumbre y tal vez también la seguridad de tener un producto final suficientemente homogéneo. Pero ¿qué sucede con la molienda conjunta? Pues, a mi entender — dijo-, lo que pasa siempre que se muelen dos materiales, uno más blando y otro más duro: que el primero se refina más que el segundo y se obtiene un producto con una curva granulométrica muy extendida, esto es, con mucha cabeza y mucha cola, por abundancia tanto de gruesos como de finos. Y esto - se preguntó-, ¿es bueno o malo, en comparación con lo que pasa en una molienda por separado, de la que resulte una mezcla de productos que en conjunto tenga una curva granulométrica más corta — con menos cabeza y menos cola-? Creo — prosiguió-, que es preferible lo segundo a lo primero, a condición de que la homogeneidad del producto final sea, cuando menos, la misma que en el caso de la molienda conjunta. A propósito de todo esto -añadió-, se podría entrar en consideraciones acerca de las necesidades de agua y de la reología de las pastas, así como de las trabajabilidades resultantes para los hormigones, etc., etc.; pero, en resumen, creo - concluyó-, que cuanto menos extendida sea (dentro de ciertos límites) la curva granulométrica de un material hidráulicamente activo, como es el cemento, cualquiera que éste sea, mejor y más beneficioso será, en principio, el comportamiento general del mismo, y eso se consigue mejor con una molienda por separado, consideraciones económicas, e incluso técnicas de otro orden, aparte.

En cuanto al otro comentario - continuó el Sr. CALLEJA-, se refiere a la cuestión planteada acerca de si las cenizas volantes añadidas al clínker en el molino de fábrica se comportan mejor o peor $-\mathrm{y}$ en todo caso de forma distinta- que las mismas cenizas 
añadidas en igual proporción y al mismo cemento, en obra. A mi juicio - dijo-, creo que puede haber diferencias en cuanto a trabajabilidad. ¿Por qué? Pues porque si la ceniza entra en el molino con el clínker, poco o mucho se modifica al molerse en una cierta medida, cambiando su granulometría y su superficie. Si no pasa por el molino, esto no sucede. Ahora bien -continuó-, entre que suceda y no suceda puede haber aún diferencias más profundas, como puede ser la de que las cenosferas a las que se refería el Sr. GARCIA CONDE en el primer Coloquio, esto es, los gránulos esféricos de productos más o menos fundidos de las cenizas, se mantengan si éstas no pasan por el molino, o se destruyan al menos en parte, si las cenizas pasan por él. Estas cenosferas - prosiguió-, se pueden comparar en cierto modo con las burbujas que introduce en el hormigón un aditivo inclusor de aire. Esto hace que entre hormigones con cenizas contenidas en el cemento - molidas - o añadidas en obra - no molidas-, puede haber diferencias en cuanto a escurrimiento, plasticidad, trabajabilidad, manejabilidad, transporte, aptitud para el bombeo, etc., a igualdad de todo lo demás, en el sentido de que probablemente será más fluido, suelto, móvil y manejable el hormigón al que se le añade la ceniza en la hormigonera, que el hormigón hecho con cemento que contiene la ceniza añadida en fábrica. Esto - dijo-, creo que responde un tanto a la pregunta hecha en su momento por el Sr. GARCIA CONDE respecto de la ventaja que supone el que las cenizas volantes contengan cenosferas.

Y no habiendo más cuestiones que tratar, el Presidente, Sr. NADAL, agradeció las actuaciones del Sr. DUTRON y de todos los participantes en el Coloquio y dio éste por concluido. 